\title{
Additive Manufacturing for Fabrication of Robotic Components
}

\author{
Wen Chen* and Shengbiao Zhang \\ Department of Mechanical and Industrial Engineering, University of Massachusetts, USA
}

*Corresponding author: Department of Mechanical and Industrial Engineering, University of Massachusetts, Amherst, MA 010032210, USA

\begin{abstract}
Additive manufacturing is a promising technology in the fabrication of robotic components, because of its capability of producing complex net-shaped parts with less working procedures in combination with material science. In this review, different kinds of robotic components produced by additive manufacturing are presented, including parts of soft robots, components of multi-materials, and unibody robotic structures.
\end{abstract}

Keywords: Additive manufacturing; Robotic components; Soft robot

\section{Introduction}

Additive manufacturing (AM) is a novel materials processing approach to create parts with three-dimensional (3D) geometries by progressively adding thin layers of materials guided by a digital model. This unique feature allows production of complex or customized parts directly from the design without the need for expensive tooling or forms such as punches, dies or casting molds and reduces the need for many conventional processing steps [1]. Nowadays this technology has become capable of producing complex net-shaped parts that can be directly used as functional components made of a variety of materials, including polymers, metals, ceramics, and composites [2]. Current additive manufacturing technologies mainly include direct laser deposition, direct ink writing, selective laser sintering, inkjet printing, and projection stereolithography (Figure 1).

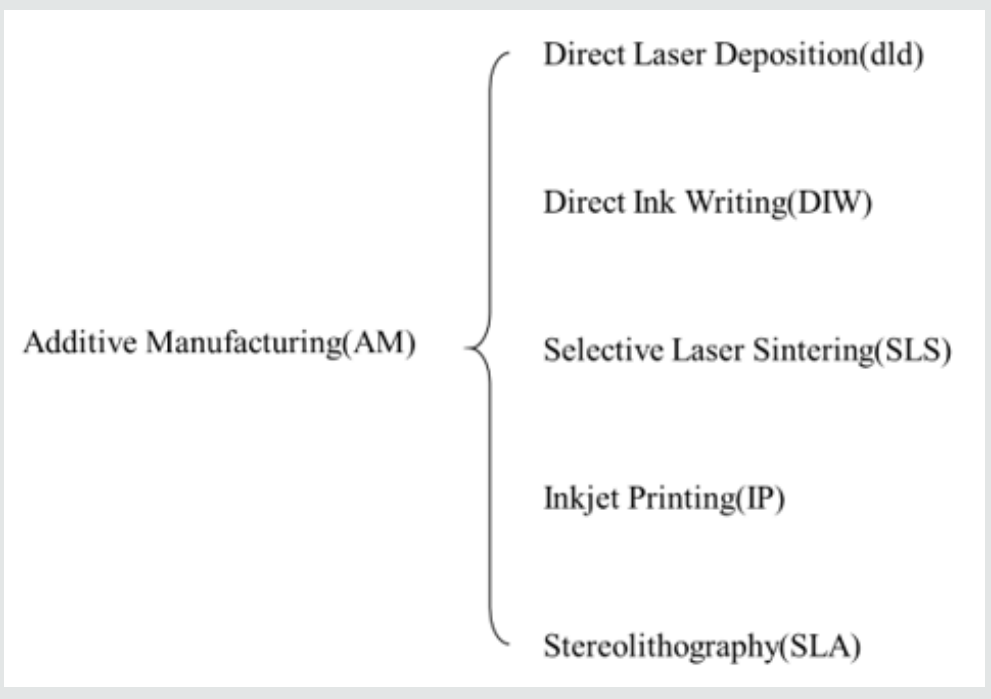

Figure 1: Different techniques of additive manufacturing. 
Robots are considered to play an important role in modern manufacturing industry. Robots and humans are expected to work hand in hand, so to speak, on interlinking tasks and using smart sensor human-machine interfaces [3]. Soft robotics combines compliant machinery and prime movers to enable continuous movement and equal force distribution that are widely used for clinic treatments. Two main benefits of soft robotics are the safety for both the robot and the human, because of a reduced stress concentration, and simple fabrication using additive manufacturing, which allows for the design of sophisticated motion and chemical manipulation [1].

\section{Additive Manufacturing for Robotic Components Soft robots}

It is very important to combine safe biological tissue with appropriate manufacturing process, which defines the key material properties in soft robots. Specifically, those properties include stiffness (k), tangent Young modulus (E), which is the tangent modulus in the linear elastic regime (usually strain $\gamma<2 \%$ ). However, there are still other mechanical features that play a significant role in the function of soft robots apart from softness, such as the ultimate elongation ( $\gamma$ ult), defining the maximum bound of components before failure, toughness $(\Gamma)$, and modulus of resilience (Ur). The material is expected to have special properties for specific robotic parts. Appropriate E, sufficient $\gamma$ ult, $\Gamma$ and Ur are helpful in large strain recovery, and high resistance to fatigue. Polymers with a wide range of physical properties are desirable in this case. The additive manufacturing of polymers involves the transition from a highly melted state to a solid network, accompanied by a rapid increment in the relative strength of the interchain interactions. It has been demonstrated that polyurethanes, silicones, and hydrogels are all printable and excellent in appropriate mechanical properties after $3 \mathrm{D}$ printing $[4,5]$.

\section{Multi-material components}

Additive manufacturing enables the integration of dissimilar materials and custom production of electromechanical devices and structural electronics. Fiber Encapsulation Additive Manufacturing (FEAM) is a multi-material additive manufacturing process in which fibers are directly encapsulated within an extruded fluid such as molten thermoplastic during printing [6]. Sensors, actuators, circuitry, and fiber-reinforced structures can be produced by FEAM, which has successfully created coils and electrical contacts, as it is capable of printing simultaneously thermoplastic polymers and conductive fillers, sometimes in combination with thermoplastic elastomers [7]. Türk D, et al. [8] investigated how additive manufacturing can support the production of composite parts along the whole process chain ranging from tooling to post processing. In this context, a novel manufacturing process route of binder jetting is also promising to produce a water-soluble sand core for hand layup of autoclave prepreg composites [8]. The novel approach is applied to a hollow high-performance robotic part, with weight savings of $54.3 \%$ compared to an advanced aluminum robotic part [9]. Ideally, one entire robot can be created using one printer, with no further required post-processing. Nevertheless, by providing automatic insertion of components and materials that are difficult or impossible to fabricate in situ, additive manufacturing allows to in situ process integrated circuits, batteries, and precision mechanical components [6].

\section{Unibody robotic structure}

Some robotic components, such as hydraulic components, may have complex structures with numerous processing steps, highly increasing the manufacturing cost and time. Additive manufacturing can produce highly integrated hydraulic components with reduced weight and higher complexity when compared to traditionally manufactured manifolds. It allows fast and free production with a lightweight structure [10]. Semini C et al. [11] presented a study on the use of additive manufacturing for hydraulic components of an agile legged robot with hydraulic actuation, where aluminum alloy AlSi $10 \mathrm{Mg}$ was applied for highly integrated hydraulic manifolds. Mechanical tests turned out that there were no visual changes after applying $20 \mathrm{MPa}$ stress. Manfredi D et al. [10] showed the fabrication of components for robotic applications of lightweight structures. A lightweight finger exoskeleton was built in a single fabrication step, which suggest an effective approach towards development of a lightweight, ergonomic, and safe design that also complies with all mechanical constraints. Recently, another method was developed by Aburaia $\mathrm{M}$ et al. [12] to manufacture an industrial robot, by combining additive manufacturing technology with modern software tools for designing industrial Robot. Collectively, these prior studies have proven that additive manufacturing is highly efficient and effective in production of robotic parts, so that engineers pay more attention to the functionality of their product [12].

\section{Conclusion}

Additive manufacturing is a promising technology for the processing of various robotic components, such as soft robots, robotic parts with multi-materials, and unibody robotic structures. Robotic components can be produced more efficiently with less working procedures by additive manufacturing when compared with conventional methods. Complex structures can be created in a more flexible way in combination with different materials. It provides an opportunity to integrate a network of actuators, sensors, controls and power systems into fully autonomous soft robots through a single, on-demand digital fabrication process. In the future, the design of robots will be tightly linked to advances in additive manufacturing techniques and materials science. 


\section{References}

1. DebRoy T, Wei HL, Zuback JS, Mukherjee T, Elmer JW, et al. (2018) Additive manufacturing of metallic components-process, structure and properties. Progress in Materials Science 92: 112-224.

2. Calignano F, Manfredi D, Ambrosio EP, Biamino S, Lombardi M, et al. (2017) Overview on additive manufacturing technologies. Proceedings of the IEEE 105(4): 593-612.

3. Bahrin MAK, Othman MF, Azli NN, Talib MF (2016) Industry 4.0: A review on industrial automation and robotic. Jurnal Teknologi 78(6-13): 137-143.

4. Wallin TJ, Pikul J, Shepherd RF (2018) 3D printing of soft robotic systems. Nature Reviews Materials 3(6): 84.

5. Walker S, Yirmibeşoğlu OD, Daalkhaijav U, Mengüç Y (2019) Additive manufacturing of soft robots. Robotic Systems and Autonomous Platforms pp. 335-359.

6. Saari M, Cox B, Richer E, Krueger PS, Cohen AL (2015) Fiber encapsulation additive manufacturing: An enabling technology for 3D printing of electromechanical devices and robotic components. 3D Printing and Additive Manufacturing 2(1): 32-39.
7. Saari M, Cox B, Galla M, Krueger PS, Richer E, et al. (2015) Multi-material additive manufacturing of robot components with integrated sensor arrays. In: Next-Generation Robotics II; and Machine Intelligence and Bio-inspired Computation: Theory and Applications IX 9494: 949404.

8. Türk DA, Kussmaul R, Zogg M, Klahn C, Leutenecker-Twelsiek B, et al. (2017) Composites part production with additive manufacturing technologies. Procedia CIRP 66: 306-311.

9. Türk DA, Triebe L, Meboldt M (2016) Combining additive manufacturing with advanced composites for highly integrated robotic structures. Procedia CIRP 50: 402-407.

10. Manfredi D, Ambrosio EP, Calignano F, Krishnan M, Canali R, et al. (2013) Direct metal laser sintering: an additive manufacturing technology ready to produce lightweight structural parts for robotic applications. La metallurgia italiana 10.

11. Semini C, Goldsmith J, Manfredi D, Calignano F, Ambrosio EP, et al. (2015) Additive manufacturing for agile legged robots with hydraulic actuation. In 2015 International Conference on Advanced Robotics (ICAR) pp. 123129.

12. Aburaia M, Markl E, Stuja K (2015) New concept for design and control of 4 axis robot using the additive manufacturing technology. Procedia Engineering 100(20): 1364-1369.

\section{CC) This work is licensed under Creative}

To Submit Your Article Click Here: Submit Article

DOI: $10.32474 /$ ARME.2020.02.000143

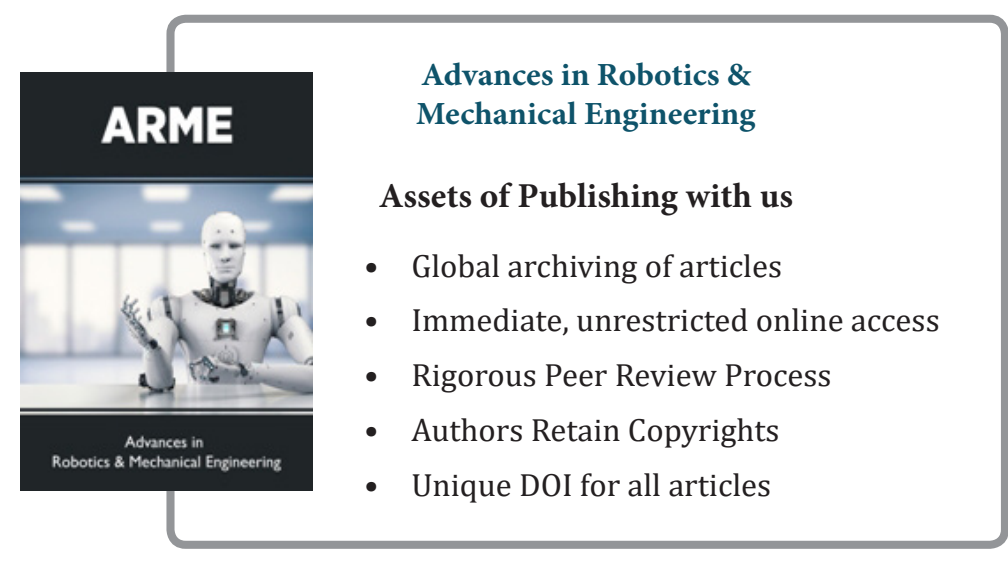

